\title{
METADATA FOR DESCRIBING EDUCATIONAL COMPETENCIES: THE UOC CASE
}

\author{
Ana-Elena Guerrero \\ Universitat Oberta de Catalunya \\ Av. Tibidabo 39-43, 08035 Barcelona, Spain \\ aguerreror@uoc.edu \\ Julià Minguillón \\ Universitat Oberta de Catalunya \\ Av. Tibidabo 39-43, 08035 Barcelona, Spain \\ jminguillona@uoc.edu
}

Keywords: E-learning, competencies, metadata, learning design, standards, LOM, IMS-LD, ontologies

Abstract: In this paper we describe a proposal for standardizing the concept of competency in a repository of learning objects, by means of an extension of the LOM and the IMS-LD standards, and the use of ontologies for ensuring consistency is also outlined. Following the recommendations from the Bologna Process, we identify the competencies required and developed by means of the current learning resources and formative and evaluation activities in a virtual e-learning environment and, using the most important taxonomies defined for such purposes in the literature, we identify the necessary metadata to describe the competencies related to such learning resources and activities. We discuss the process of shifting from content based metadata to competency based metadata, and we present an example of improving the description of learning resources by adding metadata for describing competencies, using a real case at the Universitat Oberta de Catalunya.

\section{INTRODUCTION}

Nowadays, with the creation of the new European Higher Education Area, also known as the Bologna Process, it becomes necessary to shift from heavily content-based courses to others where the concept of activity is the key. Contents, or learning resources in general, will become secondary pieces in the learning process, while the activities and the competencies developed by such activities will become the focus of any formative action. In order to do so, it is necessary to achieve an agreement for describing both contents and competencies as well, by means of metadata. It is also important to promote the formal acknowledgement of skills, knowledge and competencies gained through work experience, informal training and life experience, for prior learning recognition purposes. From the Bologna Declaration, "A Europe of Knowledge is now widely recognized as an irreplaceable factor for social and human growth and as an indispensable component to consolidate and enrich the European citizenship, capable of giving its citizens the necessary competencies to face the challenges of the new millennium, together with an awareness of shared values and belonging to a common social and cultural space", lifelong learning becomes a clear objective, using a competency-based approach for designing personalized formative itineraries. In this paper we describe a proposal for describing competencies using standardized metadata, with the aim of promoting both formative and evaluation activities based on competencies.

This paper is organized as follows: Section 2 describes the virtual e-learning environment of the Universitat Oberta de Catalunya. Section 3 reviews the concept of competency in the literature and describes the basic taxonomies adopted for metadata. In Section 4 , a proposal for the standardization of competencies through the use of metadata is outlined by extending the LOM and IMS-LD standards, and the use of ontologies is also addressed. Section 5 shows a case of study about acquiring basic competencies. Finally, the conclusions of this paper and the research lines are summarized in Section 6.

\section{THE UOC VIRTUAL CAMPUS}

The Universitat Oberta de Catalunya (Sangrà, 2002) (UOC, in English known as Open University of Catalonia) is a completely online university which offers 19 official degrees, several graduate programs and post-graduate studies, and a doctoral degree, with more than 35000 students and more than 1500 peo- 
ple including instructional designers, teachers, tutors, academic and technical staff, and so. The UOC virtual campus is an integrated e-learning environment which allows users to communicate with other users using a mail system, and includes an agenda, a news service, virtual classrooms, a digital library and other e-learning related tools. Each subject has a virtual classroom with all the needed elements for the development of the learning/teaching process: e-mail, access to documentation, the activity based teaching plan model, access to evaluation results, access to the teacher board, forums, debates, etc. The "Teaching Plan" document acts as a contract between the student and the university, and it establishes the minimum requirements (in terms of learning objectives, activities and so) that the student needs to know in order to successfully pass the subject he or she is enrolled to. This document is also a guide for helping students to prepare their learning strategy according to the planned activities during the academic semester in advance. The teaching plan is the first step towards a complete formalization of the learning process with the help of the appropriate standard descriptions. The use of e-learning standards for both content description and structuring will allow the inclusion of complex learning strategies such as personalization, for example (Mor and Minguillón, 2004). Currently, courses are structured using contents as the basic pieces for building formative itineraries, but this must be also addressed from a competency based point of view.

\subsection{From content to competencies}

In fact, both the learning materials and the teaching plan include a set of learning objectives that should be achieved by the students at the end of the academic semester. There is also a set of guided activities that students must perform in order to achieve such learning objectives. These activities have a double goal: first, they are designed to be formative activities, and second, they also serve for both student evaluation and self-evaluation. The learning objectives described there are evaluated through such activities, thus directly related to the competencies needed to achieve the desired results. Therefore, it is possible to use such information to determine the competencies required and developed by each learning resource and the associated activities. On the other hand, and from an instructional design point of view (van Merrienboer, 2001), it is necessary to integrate skills, knowledge and attitudes into professional competencies, altogether with the differentiation of various types of competencies. Content should be complemented with the appropriate metadata in order to describe such issues. Following the ideas described in (Sánchez-Alonso and Sicilia, 2005), our goal is to provide a complete description of the competencies (both required and provided by) for any learning object used to build formative itineraries fulfilling all the desired requirements.

\section{LEARNING COMPETENCIES}

Students in higher education need to acquire a set of competencies through established training actions and they have to be able to demonstrate them at the end of the course. For such purpose, it is necessary to assume a teaching and learning methodology centered in the student, where all the teaching actions are designed to promote reaching the established competency goals. Therefore, in any formative action, it will be necessary to design the content according to such purpose, as well as some activities focused towards practicing with the acquired knowledge and the reached abilities during the training activity. In order to do so, it is a key issue to identify what the competencies are, and which of them must be reached as a learning result, in order to design contents, learning resources and formative and evaluation activities.

\subsection{Basic definitions}

In general, a competency is defined as "a specific range of skill, knowledge, or ability to perform an activity". Competencies are one step beyond abilities and skills, and is the term preferred to describe combinations of attributes that describe the level or degree to which a person is capable of performing them. As it is well known, European educational institutions are evolving through the Bologna process towards a convergent European system, working together towards a new educational paradigm centered in the student, and also taking into account another goals, such the description of the qualifications in terms of load work, learning results, achieved competencies and profiles. At this stage, the concept of competency is being widely discussed, but the definition more recognized, as stated in the Tuning project report (González and Wagenaar, 2003), is already a de facto standard: "the competencies tend to convey meaning in reference to what a person is capable or competent of, the degree of preparation, sufficiency and/or responsibility for certain tasks". The complexity of subject falls in how the design of contents has to be done with respect to competencies in e-learning (Kirschner and Paas, 2001), understanding that e-learning in virtual environments is learning through Internet, which plays an important role in the delivery, support, administration and assessment of learning itself (Bates, 2000). 


\subsection{Taxonomies for competencies}

Several taxonomies for classifying competencies can be found in the literature. Among those, the wellknown Bloom's taxonomy (Bloom, 1956) classifies the educational goals and objectives on three domains: cognitive, affective and psychomotor. This taxonomy of learning behaviors can be thought of as "the goals of the training process". That is, after the training session, the learner should have acquired new skills, knowledge, and/or attitudes. Based upon these three domains, Bloom classifies the competencies that a student can reach in different levels, depending on the domain. In particular, for the cognitive domain, Bloom identifies six levels, going from a simple recall or recognition of facts as the lowest, to the highest order, which is classified as evaluation. Bloom creates relationships between competencies and demonstrated skills, in a pyramidal structure (from bottom to top): knowledge, comprehension, application, analysis, synthesis an evaluation. Each competency in the pyramid is supposed to include all the lower competencies. The first three competencies are supposed to be basic, while the acquisition of the second three competencies needs advanced skills from the student. Regarding the affective domain, Bloom defines five levels, namely: perception, response, valuing, organization and characterization. Unlike the cognitive domain, there is not a clear relationship between the levels and their degrees, although Bloom states that they follow the same pyramidal structure. Finally, as a practical guide for classifying a learning objective into a competency level, Bloom uses the verb in the learning objective as the key for classification. We have adopted this simple procedure because almost every learning resource used at UOC has one or more learning objectives described using key verbs such as identify, compare or justify, for example.

Another feasible taxonomy is the classification about competencies described in the Tuning project (González and Wagenaar, 2003). As in Bloom's taxonomy, this taxonomy states that a typical student does not either possesses or lacks a competency in absolute terms, so that competencies can be placed on a continuum. Based upon this fact, competencies are classified in two types: general competencies, which are considered important and common by everyone and to all degrees, and specific competencies that are related with a subject area and also to specific knowledge of a field of study. The general competencies are structured in three different kinds: instrumental competencies, such as elementary computing skills; interpersonal competencies, such as the ability of working in an interdisciplinary team; and systemic competencies, such as the capacity for applying knowledge practically, or the capacity to adapt to new situations. Other studies remark that the continuous evolution of learning technologies requires new competencies and the need to incorporate them, depending on the role adopted by each user at any stage of the learning process. We use the student's role as the center for the design of the competencies related to learning resources, following the UOC's pedagogical model. In any case, like some experts say, the competencies are an integrated whole of knowledge, skills and attitudes (Kirschner et al., 1997) and, as recently stated in (Reichert and Tauch, 2005), "the added value of Bologna reforms is that it offers the opportunity from concentrating more in the needs and the competencies of the students". Therefore, it is important to focus on introducing a model of teaching and learning centered on the student and based on the acquisition of competencies. For that, it is necessary to identify the general and specific competencies for each subject, and also the teaching and learning resources and activities in order to reach them. Notice that both taxonomies are not completely orthogonal, so there is some degree of overlapping. Nevertheless, both the Bloom and the Tuning taxonomies are useful for describing competencies from different points of view, thus using both of them.

\section{METADATA FOR DESCRIBING COMPETENCIES}

In this section we describe a proposal for integrating the concept of competency following the taxonomy developed in the previous section into the current e-learning standards in use at the UOC virtual e-learning environment. The description of the elements of any e-learning process and all the interactions between such elements is not a simple question. Two basic levels of description can be identified: the first level, pointed towards content management through the use of learning objects, describes the aspects directly related to the educational content. The second level describes the interactions between such learning objects and the users within the framework defined by the learning process. This separation is needed to ensure reutilization of learning resources in different contexts.

\subsection{The LOM standard}

For the first level, the LOM (Learning Object Metadata) standard defines a structure for interoperable descriptions of learning objects. In this case, a learning object is defined as any entity, digital or non-digital, that may be used for learning, education or training. Notice that we do not use the classical definition of learning object from Wiley (Wiley, 2002) because it does not include non-digital resources, which are still 
heavily used at the UOC virtual campus. Nevertheless, although there are several other definitions for learning objects, all of them coincide in a single desired behavior: reusability (Polsani, 2003).

Metadata for a learning object describes relevant characteristics of such learning object to which applies, pursuing reusability. Regarding the LOM standard, such characteristics may be grouped in nine categories, namely: general, life cycle, meta-metadata, educational, technical, educational, rights, relation, annotation, and classification. For our purposes, the "educational" and "classification" categories are the possible targets for extending the LOM standard to include descriptors about competencies, instead of the "annotation" category, which is mostly used for unstructured descriptions. Finally, although it is not necessary, it is also possible to add the appropriate data to the "meta-metadata" category to identify the source of the proposed extension.

\subsection{Extending the LOM standard}

Following the directions given in the previous sections, Table 1 resumes the new elements added to the LOM standard for describing a competency: an optional textual description and a combination of Bloom and Tuning descriptors. For each element, its name, size (or cardinality) and its type or the set of possible values are shown. The level (dependencies) of the new element is also shown, in order to clarify whether an element is present or not depending on the value of its root element. For example, both the TuningspecificLevel and TuningspecificDescription elements have only sense if a specific competency has been specified through the use of the TuningSpecificName element, within another Tuningspecific one.

Notice that we have not included metadata for the psychomotor competencies as described in Bloom (Bloom, 1956), although its implementation is straightforward following the same approach, in case of other educational environment requirements. Currently now, we have also discarded to include a confidence value as defined in (Sánchez-Alonso and Sicilia, 2005), because its usage is not clear and it might generate doubts in the teachers adopting this proposal for describing competencies. Regarding the taxonomy used for identifying the possible verbs describing cognitive competencies, it can be found in (Bloom, 1956), but it could be extended if needed.

For describing the required competencies, that is, the competencies that a student needs to perform a certain activity, they can be incorporated into the proposal using the same approach, adding a new level of description, one for required competencies ("requires") and other for developed ("develops"). These competencies identify the minimum requirements in competency terms of the learning contents used to further develop other competencies. Although required competencies are defined by instructional designers and teachers, according to their expertise, it is also possible to determine some of them from the competencies described in Table 1, using the appropriate set of rules defined in an ontology about competencies. For example, as stated in (González and Wagenaar, 2003), systemic competencies require as a base the prior acquisition of instrumental and interpersonal competencies.

Finally, for describing specific competencies directly related to narrow knowledge areas, it is better to use available taxonomies when possible. For example, for learning resources used in the Computer Science degree, the 1998 extended ACM taxonomy ${ }^{1}$ is used (at the UOC) to describe the specific content categories, while the MeSH taxonomy ${ }^{2}$ is widely used to describe medical terms. UOC is currently redefining the taxonomies for all the knowledge areas, in order to establish a common language for describing all the learning contents in the virtual campus.

\subsection{The IMS-LD standard}

The IMS-LD (Learning Design) standard tries to describe the aspects more related to the learning process in itself, such as sequencing or role playing, that is, the second level of description as aforementioned. It seems clear that this information cannot be stored in the learning objects, but in a higher semantic level. Although the IMS-LD standard may seem too complex, its flexibility and multilevel description capabilities allow the specification of any learning process ranging from simple educational itineraries to complex learning processes including personalization and collaborative working capabilities. In IMS-LD, "Learning-objectives" within an "Activity" is precisely the place to describe competencies, but using a more textual approach. Each learning objective is described using, at least, two basic fields, a text based description and a type, which can be one (and only one) of the following: skill, knowledge, insight, attitude, competency and other. Therefore, any extension to include a more comprehensive description of competencies should be included here, using the proposal presented in this paper. IMS-LD will probably become a standard for defining complex learning processes, including personalization issues, and so. Therefore, it would be interesting to study how to include our proposal in the IMS-LD standard taking also into account not only competencies but also activities and roles.

\footnotetext{
${ }^{1}$ http://www.acm.org/class

${ }^{2} \mathrm{http}: / /$ medline.cos.com/mesh/main.shtml
} 


\begin{tabular}{|l|l|c|l|}
\hline Level & Name & Size & Type \\
\hline 1 & CompetencyDescription & 0 or 1 & any text \\
\hline 2 & Bloom & 0 or 1 & - \\
\hline 2.1 & BloomCognitive & 0 or 1 & $\begin{array}{l}\{\text { knowledge, comprehension, } \\
\text { analysis, synthesis, evaluation }\}\end{array}$ \\
\hline 2.1 .1 & BloomCognitiveVerb & any & taxonomy \\
\hline 2.2 & BloomAffective & 0 or 1 & $\begin{array}{l}\{\text { perception, response, valuing, organization, } \\
\text { characterization }\}\end{array}$ \\
\hline 3 & Tuning & 0 or 1 & - \\
\hline 3.1 & TuningGeneral & 0 or 1 & $\{$ instrumental, interpersonal, systemic $\}$ \\
\hline 3.1 .1 & TuningInstrumental & 0 or 1 & $\begin{array}{l}\{\text { cognitive, methodological, technological, linguis- } \\
\text { tic }\}\end{array}$ \\
\hline 3.2 & TuningSpecific & any & - \\
\hline 3.2 .1 & TuningSpecificName & 1 & taxonomy \\
\hline 3.2 .2 & TuningSpecificDescription & 0 or 1 & any text \\
\hline 3.2 .3 & TuningSpecificLevel & 0 or 1 & $\{$ basic, medium, advanced $\}$ \\
\hline
\end{tabular}

Table 1: New metadata elements for describing a single competency.

\subsection{Integration using ontologies}

The use of ontologies for standard integration and extension is a common tool in the semantic web field. As described in (Sicilia and García-Barriocanal, 2003), it is possible to use an ontology for describing not only standards, but also the relationships that occur between the elements that take part of such standards, which cannot be part of the learning object instances, providing coherence to metadata instances and referring to the appropriate domains. The other possible use of ontologies, and perhaps more interesting, is to ensure consistency and to help users to create the metadata for a learning object, using a set of rules for both automatic metadata data filling and validation. If OWL (Web Ontology Language) (McGuinness and van Harmelen, 2004) is used for describing the ontology, it is possible to use an OWL reasoner and any other rule based language to establish all the constraints related to the LOM standard and competencies, such as RuleML or SWRL (Semantic Web Rule Language), for example. Using the latter, rules can be described as Antecedents $\Rightarrow$ Consequents, following a human readable syntaxis. For example, the fact that if a general competency is classified as one of the possible values for the instrumental category, its general category has to be specified and be indeed instrumental.

\section{EXAMPLE OF USE}

In order to exemplify the proposal described in the previous section, we provide a basic example for a learning resource about working collaboratively for searching, selecting and creating a report about one particular subject in the Computer Science degree.
This goal is structured in three continuous assessment activities and one final practical exercise, and a basic competency for making searches through the web is required (denoted by H.3.0.a in the ACM taxonomy). On the other hand, the same competency is developed altogether with others more specific about using Internet for searching, content selection and so (H.3.3).

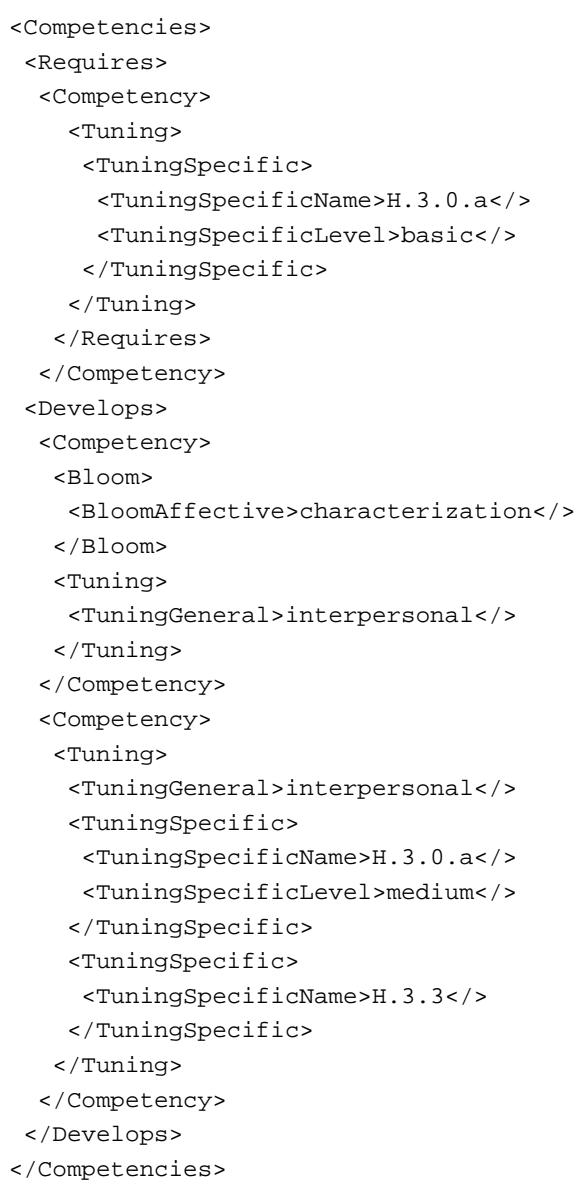




\subsection{Proposal validation}

Although the example shown above is far from being a complete proof for validating the proposal, it can be used as a basic guide for its evaluation. In fact, all the learning resources available at the UOC virtual campus are undergoing a reviewing process, including metadata for describing both content and competencies. Currently now, this represents more than one thousand text books and other learning resources which will be probably fragmented in several learning units, which will be the basic pieces of the learning objects repository. To implement this example, first it is necessary to identify the competencies required and developed in the teaching and learning for each learning resource, using learning objectives as basic sentences to determine the right category through the verbs used in each learning objective, as well as the more suitable learning resources for the design of the activities. On the other hand, for each degree and knowledge area, the specific competencies must also be identified. Finally, the use of the proposal presented in this paper arises several issues that must be addressed in order to improve the metadata that identify a learning object, and consequently, the repositories, focussing the contents, the activities and the learning resources towards the future of the European Higher Education Area, based on the acquisition of competencies. This proposal could also improve the reusability of the learning objects by means of valuable metadata.

\section{CONCLUSION}

Since the Bologna declaration in 1999, and with the appearance of the concept of competency, it becomes necessary to rethink the whole learning process from a content based point of view to another more user centered, based on activities requiring and developing competencies. In this paper we have described a proposal for standardizing the concept of competency using the LOM standard and a XML extension which can be also used in IMS-LD. The use of ontologies for both standard integration and validation and an example of usage have also been outlined.

Current and further research in this topic should include the complete description of the proposed extension using an ontology, in order to ensure a better formalization of the concept of competency and simplify the process of adding it to any other e-learning standard such as LOM and IMS-LD. The learning resources available at UOC are being currently reviewed in order to add metadata for educational purposes, so we expect to validate our proposal with a large repository of learning objects.

\section{Acknowledgements}

This work is partially supported by Spanish government grant MULTIMARK TIC2003-08064-C04-04.

\section{REFERENCES}

Bates, A. W. (2000). Managing Technological Change: Strategies for College and University Leaders. JosseyBass, Inc., San Francisco.

Bloom, B. (1956). Taxonomy of Educational Objectives, Handbook I: The Cognitive Domain. David McKay Co Inc, New York.

González, J. and Wagenaar, R. (2003). Tuning educational structures in Europe. Technical report. Available at http://www.relint.deusto.es/TuningProject/index.htm.

Kirschner, P. and Paas, F. (2001). Web-enhanced higher education: a tower of Babel. Computers in Human Behaviour, 17:347-353.

Kirschner, P., Vilsteren, P., van Hummel, H., and Wigman, M. (1997). A study environment for acquiring academic and professional competence. Studies of Higher Education, 22(2):157-171.

McGuinness, D. L. and van Harmelen, F. (2004). OWL web ontology language overview. Technical report, W3C. Available at http://www.w3.org/TR/owl-features/.

Mor, E. and Minguillón, J. (2004). E-learning personalization based on itineraries and long-term navigational behavior. In Proceedings of the Thirteenth World Wide Web Conference, volume 2, pages 264-265, NY, NY.

Polsani, P. R. (2003). Use and abuse of reusable learning objects. Journal of Digital Information, 3(4).

Reichert, S. and Tauch, C. (2005). Trends IV: European universities implementing bologna. Technical report, European University Association. Available at http://www.bologna-bergen2005.no/Docs/02EUA/050425_EUA_TrendsIV.pdf.

Sánchez-Alonso, S. and Sicilia, M. A. (2005). Normative specifications of learning objects and learning processes: Towards higher levels of automation in standardized e-learning. International Journal of Instructional Technology and Distance Learning, 2(3).

Sangrà, A. (2002). A new learning model for the information and knowledge society: The case of the UOC. International Review of Research in Open and Distance Learning, 2(2).

Sicilia, M. A. and García-Barriocanal, E. (2003). On the integration of ieee-lom metadata instances and ontologies. IEEE Learning Technology newsletter, 5(1).

van Merrienboer, J. (2001). Instructional design for competency-based learning: New directions for design, delivery and diagnosis. Interactive Educational Multimedia, 3:12-26.

Wiley, D. A. (2002). The Instructional Use of Learning Objects. Agency for Instructional Technology. 